\title{
FMRP Attenuates Activity Dependent Modifications in the Mitochondrial Proteome
}

\section{Pernille Bülow}

Emory University

\section{Peter A Wenner}

Emory University

\section{Gary J Bassell}

Emory University

\section{Victor Faundez ( $\nabla$ vfaunde@emory.edu )}

Emory University https://orcid.org/0000-0002-2114-5271

\section{Research}

Keywords: Homeostatic plasticity, FMRP, Attenuates Activity Dependent Modifications, Mitochondrial

Proteome

Posted Date: February 1st, 2021

DOI: https://doi.org/10.21203/rs.3.rs-168830/v1

License: (c) (1) This work is licensed under a Creative Commons Attribution 4.0 International License. Read Full License 


\title{
FMRP Attenuates Activity Dependent Modifications in the Mitochondrial Proteome
}

\author{
Pernille Bülow ${ }^{1}$, Peter A. Wenner*2, Gary J. Bassell ${ }^{1 *}$, Victor Faundez ${ }^{1 *}$ \\ Departments of Cell Biology ${ }^{1}$ and Physiology ${ }^{2}$. Emory University School of Medicine. Atlanta. GA \\ 30322
}

Running Title: Altered Mitoproteome in Fragile X

* Co-corresponding Authors 


\begin{abstract}
Homeostatic plasticity is necessary for the construction and maintenance of functional neuronal networks, but principal molecular mechanisms required for or modified by homeostatic plasticity are not well understood. We recently reported that homeostatic plasticity induced by activity deprivation is dysregulated in cortical neurons from Fragile X Mental Retardation protein (FMRP) knockout mice [1]. These findings led us to hypothesize that identifying proteins sensitive to activity deprivation and/or FMRP expression could reveal pathways required for or modified by homeostatic plasticity. Here, we report an unbiased quantitative mass spectrometry used to quantify steady-state proteome changes following chronic activity deprivation in wild type and $\mathrm{Fmr1}^{-/ y}$ cortical neurons. Proteome hits responsive to both activity deprivation and the $\mathrm{Fmr} \mathrm{I}^{-/ y}$ genotype were significantly annotated to mitochondria. We found an increased number of mitochondria annotated proteins whose expression was sensitive to activity deprivation in Fmr1$/ y$ cortical neurons as compared to wild type neurons. These findings support a novel role of FMRP in attenuating mitochondrial proteome modifications induced by activity deprivation.
\end{abstract}




\section{Introduction}

Homeostatic plasticity represents a set of mechanisms that act to maintain physiologically appropriate activity levels within a preset target range or set point. Following chronic activity perturbations, neurons and networks demonstrate the capacity to recover original activity levels by deploying several different adaptive mechanisms [2-6]. While these physiological/functional mechanisms have been extensively studied, far less is known about the underlying molecular cascades that sense, respond, and/or drive these changes. Alterations in cytoplasmic calcium and modifications of protein synthesis are two components of the signaling cascades that underlie these homeostatic mechanisms $[4,7,8]$. Recent evidence has implicated mitochondria as bona fide regulators of neuronal activity. First, by buffering presynaptic calcium levels, mitochondria regulate the neural activity set point for homeostatic plasticity [9]. Secondly, mitochondria provide ATP necessary to support protein synthesis during neuronal maturation and plasticity $[10,11]$. Despite these advances, principal mechanisms required for the establishment, modulation and/or maintenance of homeostatic plasticity and the role of mitochondria in this process remain poorly understood.

Several rare human genetic disorders associated with autism and other neurodevelopmental disorders also exhibit impaired homeostatic plasticity [1, 12-16]. This is evident in the case of gene defects in FMR1 and MECP2, causative of Fragile X (FXS) and Rett Syndrome, respectively, whose deficiency alters homeostatic plasticity responses $[1,12,14,17$, 18]. FMR1 and MECP2 control the expression of a vast number of genes at the translational and transcriptional levels in neurons [19-24]. This suggests that FMR1- or MECP2-dependent gene expression mechanisms likely regulate multiple factors necessary for the establishment, modulation, and maintenance of homeostatic plasticity.

Here, we sought to identify major pathways or organelles associated with changes in homeostatic plasticity expression. Since we and others reported the necessity of FMRP for the appropriate expression of homeostatic plasticity [1, 12], we hypothesized the existence of common proteins sensitive to both neuronal activity and FMRP expression. To address this hypothesis, we treated wild type and $F m r 1^{-/ y}$ mouse cortical primary neuronal cultures with tetrodotoxin (TTX) plus a NMDA receptor antagonist, and subsequently evaluated their steady state proteomes using unbiased quantitative mass spectrometry. We focused on NMDA blockade as it has been used as a common method of triggering homeostatic plasticity [25, 26]

In contrast with acute proteome labeling strategies performed on wild type neurons, such as BONCAT or SILAC [27-29], we found that following prolonged activity deprivation the mitochondrion, but not the synapse, was the most affected cellular organelle at steady state in both wild type and $F m r 1^{-/ y}$ neurons. While both wild type and $F m r 1^{-/ y}$ neurons displayed changes in the mitoproteome following activity deprivation, these responses were exaggerated in $\mathrm{Fmr1}^{-}$ Iy neurons. Our findings support the idea that mitochondria are modified by activity 
perturbations, and possibly mediate aspects of homeostatic plasticity expression. We postulate that mitochondria are targets of genes causative of neurodevelopmental disorders and suggest that mitochondrial plasticity defects may contribute to neurodevelopmental disorders and their comorbidities. 


\section{Materials and Methods}

\section{Experimental model and subject details}

Mice: FMR1 ${ }^{\text {HET }}$ females (backcrossed on C57BL6 background, B6.129P2-Fmr1tm1Cgr/J Stock No: 003025) were crossed with WT C57BL6 males (Jackson Laboratory) to generate litters of pups with mixed genotypes (Fmr1 ${ }^{-1 y}$, Fmr1 ${ }^{\text {HET }}$ or wild-type (WT)). Thus, for all experiments, $\mathrm{Fmr1}^{-1 \mathrm{y}}$ male pups were compared to their WT littermate control. We performed PCR to identify genotypes on postnatal day $0-1$ (P0-P1) as described previously $[30,31]$. The mice were housed in a $12 \mathrm{hr} \mathrm{light/dark} \mathrm{cycle} \mathrm{and} \mathrm{the} \mathrm{animal} \mathrm{protocol} \mathrm{was} \mathrm{approved} \mathrm{by} \mathrm{the} \mathrm{Institutional} \mathrm{Animal} \mathrm{Care}$ and Use Committees at Emory University.

Primary cortical neuronal cultures: Cerebral cortices were dissected and cultured from genotyped wild type and $\mathrm{Fmr}^{-1 /}$ pups on PO-P1. The cortices were enzymatically dissociated using trypsin (Thermo Fisher Scientific; 10 min), mechanically dissociated in Minimum Essential Media (MEM; Fisher) supplemented with 10\% Fetal Bovine Serum (FBS; Hyclone) and stained to assess viability using Trypan Blue (Sigma). 100,000 neurons were plated on $35 \mathrm{~mm}$ Glass Bottom MatTek petri-dishes (MatTek Corp., Cat no: P35G-1.5-14-C) coated with FBS (Gibco), poly-lysine (Sigma) and laminin (Sigma). The neurons were cultured in standard growth medium (glial conditioned neurobasal (Fisher) supplemented with glutamax (Gibco) and B27 (Invitrogen)), and half of the media was exchanged 2-3 times a week until experimental treatments began. No antibiotics or antimycotics were used. The cultures were maintained in an incubator regulated at $37 \mathrm{C}, 5 \% \mathrm{CO}_{2}$ and $95 \%$ relative humidity. Culturing protocol is similar to Bülow et al. [1]. All experiments were performed with days in vitro (DIV) 12 neuronal cultures.

\section{Methods detail}

Proteomics: Cell cultures were placed on ice, washed thrice with PBS and lysed with a 8M Urea $(\mathrm{pH}$ 8.5). The urea lysis buffer included an EDTA free HALT protease and phosphatase inhibitor cocktail (Thermo Fisher Scientific, cat no.:78441). The samples were immediately placed on dry ice, and kept at $-80 \mathrm{C}$.

Protein Extract Preparation and Digestion. Lysates were quantified by Qubit fluorometry (Life Technologies), $50 \mu \mathrm{g}$ of each sample was digested overnight with trypsin for $1 \mathrm{~h}$ at room temperature in $12 \mathrm{mM}$ DTT followed by alkylation for $1 \mathrm{~h}$ at room temperature in $15 \mathrm{mM}$ iodoacetamide. Trypsin was added to an enzyme : substrate ratio of 1:20. Each sample was acidified in formic acid and subjected to solid phase extraction on an Empore SD C18 plate (3M 
catalogue\# 6015 SD). Each sample was lyophilized and reconstituted in 140mM HEPES, pH 8.0, $30 \%$ acetonitrile for TMT labeling.

TMT Labeling. $40 \mu \mathrm{L}$ of acetonitrile was added to each TMT tag tube and mixed aggressively. Tags were incubated at RT for $15 \mathrm{~min}$. $15 \mu \mathrm{L}$ of label was added to each peptide sample and mixed aggressively. Samples were incubated in an Eppendorf Thermomixer at 300 $\mathrm{rpm} 25^{\circ} \mathrm{C}$ for $1.5 \mathrm{~h}$. Reactions were terminated with the addition of $8 \mu \mathrm{L}$ of fresh $5 \%$ hydroxylamine solution and $15 \mathrm{~min}$ incubation at room temperature. Each labeled sample was combined into two experiments, frozen, and lyophilized and subjected to SPE on a High-Density 3M Empore SDB-XC column (Cat. \#4340-HD). The eluent was lyophilized, resuspended and subjected to high $\mathrm{pH}$ reverse phase fractionation in a XBridge C18 colum (Waters, part \#186003023) on a Agilent $1100 \mathrm{HPLC}$ system equipped with a $150 \mu \mathrm{L}$ sample loop operating at $0.3 \mathrm{~mL} / \mathrm{min}$, detector set at $214 \mathrm{~nm}$ wavelength.

Mass Spectrometry. Peptides were analyzed by nano LC/MS/MS with a Waters NanoAcquity HPLC system interfaced to a ThermoFisher Fusion Lumos mass spectrometer. Peptides were loaded on a trapping column and eluted over a $75 \mu \mathrm{m}$ analytical column at $350 \mathrm{~nL} / \mathrm{min}$; both columns were packed with Luna C18 resin (Phenomenex). Each high pH RP pool was separated over a $2 \mathrm{~h}$ gradient ( $24 \mathrm{~h}$ instrument time total). The mass spectrometer was operated in data-dependent mode, with MS and MS/MS performed in the Orbitrap at 60,000 FWHM resolution and 50,000 FWHM resolution, respectively. A 3s cycle time was employed for all steps.

Data were processed through the MaxQuant software v1.6.2.3, which served for recalibration of MS data, filtering of database search results at the $1 \%$ protein and peptide false discovery rate (FDR), calculation of reporter ion intensities (TMT), isotopic correction of reporter ion intensities (TMT). Data were searched using Andromeda with the following parameters: Enzyme: Trypsin, Database: Swissprot Mouse, Fixed modification: Carbamidomethyl (C), Variable modifications: Oxidation (M), Acetyl (Protein N-term), Fragment Mass Tolerance: 20ppm. Pertinent MaxQuant settings were: Peptide FDR 0.01, Protein FDR 0.01, Min. peptide Length 7, Min. razor + unique peptides 1, Min. unique peptides 0, Second Peptides FALSE, Match Between Runs FALSE. The proteinGroups.txt file was uploaded to Perseus v1.5.5.3 for data processing and analysis. We considered proteins hits as such if the fold of change was above or below (average of all hits in the dataset $) \pm(2 S D)$ with an alpha< 0.05 as previously described $[32,33]$.

The mass spectrometry proteomics data have been deposited to the ProteomeXchange Consortium via the PRIDE [34] partner repository with the dataset identifier PXD021473 and 10.6019/PXD021473.

Pharmacology:_Drugs were used in the following concentrations (in $\mu \mathrm{M})$ : TTX, 1 (Tocris); APV, 100 (Tocris). Drugs were added to fresh standard growth medium and added to the cultures 
by a complete media change on Day in vitro (DIV) 10 and lasted for 48 hours. The treatment drugs were refreshed after 24 hours. Control cultures had a simultaneous complete media change but without drugs. Cultures were randomly assigned to each treatment group (control, TTX/APV). All experiments occurred on DIV 12.

Ontology Analyses: We used ENRICHR and ClueGo gene ontology tools to identify annotated cellular compartments as previously described $[35,36]$. We used as cutoff for inclusion $\mathrm{p}$ values $<0.05$. Raw data are presented in Extended Data Table 2.

Statistics: Two tailed statistical tests were performed as indicated in figure legends using Kaleida Graph 4.5.2 and Aabel NG2 v5.20. No outlier data curation was applied to statistical analyses. 


\section{Results}

\section{The Neuronal Proteome is Sensitive to Activity Blockade.}

We used 12 days in vitro (DIV) primary neuronal cultures from wild type and $F m r 1^{-1 y}$ neurons, and treated them with tetrodotoxin (TTX) and the NMDAR receptor antagonist (2R)amino-5-phosphonovaleric acid (APV) for 48 hours to induce homeostatic plasticity [1]. To identify proteomic changes affecting whole neurons instead of proteome modifications focalized to the synapse, we focused on steady state changes in the proteome after prolonged changes in neuronal activity. We used Tandem Mass Tagging (TMT) quantitative mass spectrometry to measure proteome modifications [37]. Our steady-state experimental design differs from previously used mass spectrometry approaches where synaptic effects of either activity deprivation or FMRP expression are better revealed with acute proteome labeling [27-29, 38, 39]. In contrast, TMT labeling offers insights into global and long-lasting proteomic changes induced by activity deprivation and FMRP loss. Therefore, the results obtained here to investigate how steady state changes in the $F m r 1^{-/ y}$ proteome might differ from that obtained to measure the dynamics of the newly synthesized proteome and responses to mGluR activation in Fmr1-/y cells [38].

We quantified 6,074 proteins in a 22-plex experimental design to simultaneously compare wild type and $F m r 1^{-1 / y}$ neuronal cultures treated with vehicle or TTX-APV (Fig. 1A-C). The proteome discriminated cultures by their FMRP protein expression (Fig. 1C, upper panels blue square symbols). Wild type and $F m r 1^{-1 /}$ proteomes were largely similar at baseline except for the expression of a discrete number of non-overlapping proteins (Fig. 1C, upper panels). Principal component analysis (Fig. 1D) and similarity matrix comparisons (Fig. 1 E) confirmed the resilience of the steady state proteome to Fmr1 gene defects. Fmr1-/y effects on the proteome were discrete; the expression of only 14 proteins was sensitive to genotype, or $0.23 \%$ of the quantified proteome. This result may seem to diverge from the conventional idea that loss of FMRP leads to elevated global protein synthesis, which is often directly measured with acute labeling strategies [38, 40,41]. However, although a large number of FMRP target mRNAs have increased efficiency of translation, very few of these result in noticeable differences in protein expression at steady state in FMRP deficient neurons [42]. This discrepancy between steady state and acute/dynamic protein levels has been ascribed to increased rates of protein degradation [31] in concurrence with increased protein synthesis. Thus, while steady state levels may appear similar, synthesis and degradation rates of FMRP-targeted mRNAs may differ substantially [42]. The small proteomic difference between genotypes was maintained after TTX/APV treatment: only 23 proteins, or $0.38 \%$ of the quantified proteome, differed when comparing activity deprived wild type and $\mathrm{Fmr1}^{-/ y}$ neurons (Fig. 1C, F, and G p=0.138, z-score 1.48). Notably, the 6,074 quantified proteins poorly overlapped with the FMRP bound mRNAs curated from publicly available 
independent datasets by Suhl et al. (Fig. 1H) [43]. Only Fam120a was shared between our dataset and the 568 gene set curated by Suhl et al (Fig. $1 \mathrm{H}$ ). This suggests that the minimal genotype effect in proteome composition may be biased by the low detectability of direct FMRP-binding targets in our dataset.

In contrast with the genotype effects, the most pronounced proteome modifications happened after TTX-APV treatment. The TTX-APV effect occurred both in wild type and mutant cells (Fig. 1C lower panels and F). We identified 159 proteins whose expression changed in wild type cultures after TTX-APV treatment (Fig. 1C lower panels and F, and Extended Data table 1). Strikingly, treatment-dependent proteome alterations were significantly more pronounced in $\mathrm{Fmr1}^{-/ y}$ cells where we identified a significantly higher number of proteins whose expression was sensitive to TTX-APV treatment: in total 260 proteins were changed in $\mathrm{Fmr1}^{-/ y}$ cells after TTX/APV (Fig. 1C lower panels, F, and G p<0.0002 and z-score of 5.02, and Extended Data table 1). The TTXAPV-induced proteome modifications in wild type and $F m r 1^{-1 / y}$ neuronal cultures were mostly overlapping (Fig. 1C upper right panel). In fact, $\sim 67 \%$ of all proteins sensitive to TTX-APV treatment in wild type cultures were also among the TTX-APV-sensitive proteins in Fmr1 ${ }^{-/ y}$ cells (Fig. 1F, 106 protein overlap, and Extended Data table 1). This overlap is 15.5 times higher than expected by chance between these two conditions $(p=6.67 e-114$, Exact hypergeometric probability). Conversely, $\sim 33 \%$ of the proteomes sensitive to TTX-APV differed between genotypes. However, gene ontology analysis of this diverging proteome did not identify ontologies unique to TTX-APV treated $\mathrm{Fmr1}^{-/ y}$ cells. Unsupervised clustering analysis of the whole proteome confirmed that the main variable segregating proteomes was TTX-APV treatment instead of genotype (Fig. 1E). Genotype and TTX-APV treatment did not change the proportion of neurons and astrocytes in cultured cells as assessed by the expression of neuronal and glial enriched markers (Fig. 2) [44]. These findings demonstrate that TTX-APV treatment preferentially drives proteome modifications in wild type and mutant cultures. However, more proteins were dysregulated following activity deprivation in $\mathrm{Fmr1}^{-/ / y}$ cells.

\section{Mitochondrial Proteins are Enriched in the Proteome Sensitive to Activity Blockade in Wild Type and Fmr1 ${ }^{-/ y}$ Neurons.}

We used orthogonal bioinformatic tools to identify pathways and compartments enriched from the hits identified in our proteome datasets. Due to the small size of the genotype-sensitive proteome and the large overlap in TTX-APV sensitive proteome between genotypes, we hypothesized that wild type and $F m r 1^{-1 /}$ neurons could engage similar activity dependent pathways. We first interrogated our datasets for enrichments in proteins annotated to synaptic compartments and processes using the SynGo knowledgebase [45]. The 6,074 proteins quantified in the neuronal cultures significantly enriched pre- and post-synaptic annotated 
proteins (GO:0098793 and GO:0098794, p= 5.0E-25 and 1.4E-32, FDR corrected p-value, Fig. 3A). However, the TTX-APV sensitive proteomes did not enrich synaptic annotated genes in either wild type or Fmr1-/y cells (Fig. 3A). This lack of synaptically annotated genes is comparable to the enrichment obtained with an identically sized randomly generated gene dataset (Fig. 3A). In contrast, the SFARI annotated autism spectrum gene set, the Human Phenotype Ontology autism annotated genes (HP_0000729) [46], as well as the curated list of FMRP-binding mRNAs, all significantly enriched genes annotated to the synapse by the SynGo knowledgebase (Fig. 3A). These findings argue that steady state TTX-APV-dependent changes of the proteome involve compartments other than the pre- and post-synapse.

We used the ENRICHR and ClueGo gene ontology tools to identify annotated cellular compartments within the proteome affected by the TTX-APV treatment in both wild type and $\mathrm{Fmr1}^{-1 / y}$ cultures $[35,36]$. We predicted that because of the significant overlap between the Fmr1/y and wild type TTX-APV proteome data sets (Fig 1F), they should encompass shared ontologies. We further predicted that pooling together proteins sensitive to TTX-APV in wild type and Fmr1${ }^{\prime} y$ cultures should additionally enrich relevant cellular compartment ontologies despite increasing dataset size [47]. Conversely, a pooled dataset should decrease the significance of annotated compartments marginally represented in the TTX-APV wild type and TTX-APV Fmr1-/y datasets [47]. The only annotated terms that satisfied these criteria were the mitochondrion and dendrite (Fig. 3B compare red, black and green bars. GO:0005739, $p=1.22 \mathrm{E}-04$ and GO:0030425, $p=2.88 \mathrm{E}-$ 04, and Extended Data Table 2-1). Mitochondrial and dendrite annotated terms were also identified with the ClueGo algorithm (Fig. 3C-E. GO:0031966, mitochondrial membrane, $p=$ 0.0013. GO:0030425, dendrite, $p=0.0033$, and Extended Data Table 2-1). Among these compartments, the mitochondrial annotated terms respiratory chain and oxidoreductase complex were similarly represented in each of the TTX-APV-sensitive individual and pooled datasets (Fig. 3D-E, GO:0098803 and GO:1990204, p=0.048 and 0.046, and Extended Data Table $2-1)$. These results show that mitochondria are robustly identified as compartments affected by TTX-APV in wild type and Fmr1-/y primary neuronal cultures.

We next addressed the quality and magnitude of protein changes affecting mitochondria after TTX-APV in wild type and $F m r 1^{-/ y}$ cells. Our proteome covered $\sim 63 \%$ of all mitochondrial proteins annotated by Mitocarta (Fig. 4A) [48]. The number of mitoproteome hits observed in wild type neurons treated with TTX-APV (11 hits) were increased in the Fmr1/y cells (26 hits) (Fig. $1 C-G, 3 B-E, 4 B-E)$. Among the Mitocarta annotated proteins, 34 were sensitive to treatment with TTX-APV in either wild type or Fmr1 ${ }^{-1 y}$ cells (Fig. 1C yellow symbols and $4 \mathrm{~A}-\mathrm{C}$ ). This overlap is 1.8 times higher than expected by chance. The 34 TTX-APV sensitive proteins displayed complex changes in expression: 12 proteins were upregulated after TTX-APV while 22 were downregulated (Fig. 4C, see Expanded data Table 1). Of these 34 proteins sensitive to TTX-APV 
treatment, 11 significantly changed in similar magnitude and direction both in wild type and Fmr1 $1^{-1 y}$ cells treated with TTX-APV (Fig. 4B-D, see Fig. 4C, see Extended data Table 1). The 34 mitochondrial proteins sensitive to TTX-APV in both genotypes could be assembled into a REACTOME pathway network dominated by the citric acid (TCA) cycle and respiratory electron transport Homo sapiens annotated term (Fig. 4E, R-HSA-1428517, p=2.05E-7) [49]. However, this functional pathway network included proteins up- and down-regulated after TTX-APV treatment (Fig. 4E, teal and gray nodes, respectively) precluding precise metabolic hypothesis formulation from these changes in the mitochondrial proteome.

Since mitochondrial ontologies were revealed by their TTX-APV treatment rather than the Fmr1 genotype, we further inquired whether mitochondrial genes were annotated either to autism spectrum disorder curated databases or whether mitochondrial nuclear encoded mRNAs were bound by FMRP. Only two Mitocarta genes were present in the HPO annotated autism spectrum disorder term, an overlap 10 times below expected (Fig. 4F, HP_000072) [46]. Moreover, only 16 mRNAs annotated to Mitocarta were targets of FMRP, an overlap 2-fold lower than expected (Fig. 4G, $\mathrm{p}<7.6 \mathrm{e}-04$ ). These results suggest two different possible models. First, Fmr1 mutations and other autism spectrum disorder gene defects may indirectly regulate mitochondrial protein composition at steady state. Since FMRP targets are known to be linked to synapse function, and synapses regulate mitochondria function, the dysregulation we observe in mitochondrial proteome in $F m r 1^{-1 y}$ cells, could be downstream of synaptic FMRP targets. Alternatively, Fmr1 gene defects may directly affect mitochondria, but are below detection and/or exist in a compartment specific manner rather than by globally altering mitochondrial composition in neurons. Compartmentally localized mitochondrial changes could evade detection by mass spectrometry analysis of whole culture lysates. 


\section{Discussion}

Here, we sought to identify novel mechanisms required or modified during the induction, establishment and/or maintenance of homeostatic plasticity. To this end, we comprehensively and unbiasedly quantified steady state proteomic modifications in wild type and $\mathrm{Fmr}^{-/ \mathrm{y}}$ mouse primary cortical neurons following activity deprivation with TTX-APV (48 hr, Fig. 1A). We selected TTX-APV and FMRP expression because we previously showed that this drug treatment induced homeostatic intrinsic plasticity in a functionally altered manner in $F m r 1^{-/ y}$ neurons [1]. We draw two conclusions: first, the mitochondrial proteome is the most affected during chronic activity deprivation in a steady-state analysis. This finding represents a conceptual advance since a link between homeostatic intrinsic plasticity and the mitoproteome has not been previously shown. Second, Fmr1 $1^{-1 y}$ neurons display exaggerated changes in the mitoproteome during activity deprivation. Thus, FMRP attenuates activity dependent modifications of the mitoproteome.

How does FMRP deficiency trigger enhanced changes in the mitochondrion proteome during TTX/APV? We speculate that FMRP does so by an indirect mechanism. This assertion is founded on the marked underrepresentation of the mitochondrial proteome among the nuclear encoded mRNAs bound and translationally regulated by FMRP (Fig. 4G). In fact, only 16 of the 1158 Mitocarta annotated mitochondrial proteins are FMRP targets [19-21, 43, 50]. While this is an small number, these 16 proteins could have widespread impacts in the mitochondrial proteome. An interesting candidate for an indirect FMRP-dependent mechanism controlling the mitochondrial proteome is the mitochondrial ribosome [51]. We recently found that downregulation of mitochondrial ribosome subunits phenocopies mutant dFmr1 phenotypes in Drosophila synapses [52-54]. We posit that this mechanism is indirect because none of the 80 mitochondrial ribosome mRNAs are among the curated FMRP targets [43]. mRNAs encoding mitochondrial ribosome subunits are increased in $\mathrm{FMR1}^{-/ y}$ neuronal cells, yet their translational efficiency is decreased [51]. FMRP is best known as a translational suppressor [41], but it also regulates protein conformation/function directly or activates mRNA translation [55, 56]. FMRP can also directly bind to the cytoplasmic ribosome affecting the expression of mitochondrial proteins encoded in the nuclear genome [57]. Any of these mechanisms could indirectly impair the function and/or the composition of the mitochondrial ribosome. A compromised mitochondrial ribosome could account for altered mitochondrial functions observed in $\mathrm{FmrI}^{-/ \mathrm{y}}$ cultures [51, 58-65]. Alternatively and/or in addition, we know that loss of FMRP dysregulates synaptic and ion channel protein expression at baseline, and these alterations may lead to altered mitochondrial plasticity during activity deprivation. Since FMRP directly regulates the synaptic proteome via translational control, and directly regulates ion channel function through RNA and 
protein associations, this opens up numerous possibilities on the underlying mechanism for altered mitochondrial plasticity in FXS.

We focused on the consequences of prolonged activity deprivation on the steady state proteome. Our findings complement proteome analyses studying protein synthesis and half-life in primary cultured neurons using non-equilibrium proteome labeling strategies, such as BONCAT or isotopic labeling of neurons or neuronal fractions either by pulsed methionine or SILAC [27$29,38,39]$. SILAC and BONCAT acute labeled proteomes are enriched in synaptic proteins, a fact that highlights the responsiveness of the pre- and postsynaptic proteome to rapid changes in neuronal activity. In contrast, our findings indicate that the presynaptic and postsynaptic proteomes at the steady state remain mostly unchanged after prolonged activity deprivation both in wild type and Fmr1 ${ }^{-/ y}$ neurons (Fig. 4). Our activity-dependent steady-state proteome is enriched in proteins annotated to mitochondrial ontologies (Fig. 4B-E). The apparent discrepancy between the results obtained with acute labeling of the proteome and our steady state changes cannot be attributed to an underrepresentation of the synaptic proteome in our dataset, as they were significantly enriched (Fig. 4A). Thus, we postulate that the following three variables could contribute to the ontological differences between acute and steady-state proteomes after activity deprivation. First, the duration or the pharmacological agents used to achieve activity deprivation could underlie differences. We used 48 hours of TTX-APV treatment in contrast with the 24 hours of TTX treatment used for acute proteome labeling [27, 29]. This time difference may play an important role as the half-life of synapse annotated proteins is $\sim 5$ days, while proteins annotated to the mitochondria possess a half-life between $~ 9-15$ days [28]. Thus, acute labeling strategies are more likely to identify changes in the synaptic proteome and other cellular compartments with shorter half-lives. Further, the addition of an NMDA receptor blocker could contribute to differences as this is known to be critical for the induction of homeostatic plasticity $[25,26]$. A second variable is the difference in neuron species. While we used postnatal mouse cortical neurons, others have used postnatal hippocampal rat neurons [27-29, 47, 66]. Finally, compartment-specific mitochondrial responses could produce differences. This hypothesis is supported by evidence indicating that changes in mitochondrial protein expression following depolarization can only be detected in synaptic-enriched fractions [39]. We postulate that our activity- and Fmr1-dependent steady-state proteome reflects long-lived compartmental changes rather than rapid activity-dependent remodeling of acute proteome labeling. Further, we hypothesize that FMRP plays an important role in attenuating the activity deprivation induced changes in the mitochondrial proteome. 


\section{Declarations:}

\section{Ethics approval and consent to participate}

Not Applicable

\section{Consent for publication}

All Authors concur with publication of this manuscript.

\section{Availability of data and materials}

The mass spectrometry proteomics data have been deposited to the ProteomeXchange Consortium via the PRIDE with the dataset identifier PXD021473 and 10.6019/PXD021473.

\section{Competing interests}

There are no interests to declare by all authors

\section{Funding}

This work was supported by NIH grants 1R01MH109026 (GJB), R01NS065992 (PAW), 1RF1AG060285 (VF).

\section{Authors' contributions and Acknowledgements}

PB, GJB, PAW and VF conceived of the experimental design. PB performed all experiments. VF performed bioinformatic analysis. SZ performed Seahorse oximetry assays and data analysis. PB and VF wrote the manuscript. All co-authors contributed with comments and edits to the manuscript. 


\section{Figure Legends}

\section{Figure 1 The Neuronal Proteome Sensitive to Activity Deprivation and Fmr1-/y.}

A) Diagram of the experimental design.

B) Representative Coomassie and FMRP-beta actin blots of cultured neuronal extracts.

C) Volcano Plots of TMT mass spectrometry of wild type and Fmr1 $1^{-/ y}$ neurons (DIV12) incubated in vehicle or TTX-APV for 48 hours. $\mathrm{N}=6$ wild type and $\mathrm{n}=4 \mathrm{Fmr} \mathrm{1}^{-1 / y}$ for vehicle treated cultures, $n=5$ cultures for each genotype treated with TTX-APV. Blue symbols represent downregulated proteins, red symbols upregulated proteins. FMRP is marked by dark blue and crossed square symbol. Yellow symbols depict mitochondrial proteins significantly changed.

D) Principal component analysis of the 6074 TMT protein quantifications. Note close grouping of all conditions.

E) Similarity matrix of wild type and $F m r 1^{-/ y}$ neurons incubated in the presence of vehicle or TTX-APV for 48 hours. Kendall Tau clustering analysis. Note the clustering based on TTX-APV treatment.

F) Venn diagram of common hits in wild type and $F m r 1^{-/ y}$ neurons incubated in vehicle or TTX-APV for 48 hours. Parentheses represent total number of proteins significantly modified by treatment and/or genotype. Most protein changes occur after TTX-APV treatment irrespective of genotype.

G) Fmr1 $1^{-/ y}$ neurons incubated with TTX-APV for 48 hours significantly increase the number of hits as compared to TTX-APV-treated wild type neurons. P value and $Z$ score analysis of the number of hits in $F$. $X$ and $Y$ axes numbers represent the number of hits in parentheses in $F$ ). Compare wild type TTX-APV (159) to Fmr1 ${ }^{-/ y}$ TTX-APV (260), $p<0.0002$ and z-score 5 . Circle size and color denote $\mathrm{p}$ or z-score values. Values calculated with the Vassar's Difference between Two Independent Proportions tool.

H) Venn diagram all proteins quantified by TMT overlapping with curated the FMRP target mRNAs curated by Suhl et al. 2014.

See Extended Data Table 1.

Figure 2 Neuronal and Glial Markers after Activity Deprivation and Fmr1 Mutation .

A) Ven Diagram of overlaps between either neuronal or glial markers with all TMT quantified proteins (6074), protein hits sensitive to TTX-APV in wild type cells (159) or Fmr1-/y cells (260). Neuronal and glia enriched expressed genes were defined according to Cahoy et al. 2008

B) Violin plots depict TMT normalized expression of most abundant neuronal and astrocyte-enriched proteins in cultures treated with vehicle or TTX-APV both in wild type and $\mathrm{Fmr1}^{-/ y}$ genetic backgrounds. 
Figure 3 Ontological Analysis of the Proteome Sensitive to Activity Deprivation and Fmr1 $1 / y$.

A) Synaptic Ontology Analysis using the SYNGO tool. Sunburst plots represent synaptic annotated GO BP ontologies. Colors represent -log10 FDR corrected $p$ values. Gene sets are defined in Fig. 1. SFARI correspond to the autism spectrum disorder genes curated by https://gene.sfari.org/database/human-gene/. HP-0000729 corresponds to the Human Phenotype Ontology annotated term Autistic behavior HP:0000729 (https://hpo.jax.org/app/browse/term/HP:0000729).

B) GO CC Term ontology analysis performed with the ENRICHR tool. All proteins sensitive to TTX-APV plus those sensitive to the $F m r 1^{-/ y}$ genotype (red bars) were compared to wild type TTX-APV treated hits (black bars) and $F m r 1^{-/ y}$ TTX-APV treated hits (green bars).

C) ClueGO Ontology Analysis of all TMT Hits defined by the red bar in B. Node size represents number of mapped genes.

D) ClueGO Ontology gene percentage contributions among the three gene sets in $B$

E) ClueGO Ontology Statistics. Non-corrected Two Sided Hypergeometric Test.

See Extended Data Table 2.

Figure 4 The Mitoproteome Sensitive to Activity Deprivation and Fmr1/y.

A) Venn diagram comparing all Mitocarta 2.0 entries with all TMT quantified proteins (6074), all hits sensitive to TTX-APV plus those sensitive to the $F m r 1^{-/ y}$ genotype (335)

B) Venn diagram comparing all Mitocarta 2.0 mitoproteome with protein hits sensitive to TTX-APV in wild type cells (159) or Fmr1 ${ }^{-/ y}$ cells (260) and all TMT proteome hits (335)

C) Hierarchical clustering analysis of the expression of 34 proteins in wild type and Fmr1/y cells treated with vehicle or TTX-APV. Kendal Tau clustering analysis of rows.

D) Normalized TMT expression of top mitochondrial hits. Kruskal-Wallis Test followed by Mann-Whitney $U$ Test. $n=6$ wild type and $n=4 F m r 1^{-/ y}$ for vehicle treated cultures, $n=5$ for each genotype treated with TTX-APV TTX-APV-treated.

E) Interactome of the TTX-APV and $F m r 1^{-1 / y}$ sensitive mitoproteomes. Blue nodes represent upregulated proteins. Grey represent downregulated proteins.

F) Venn diagram of all TMT hits, the Mitocarta 2.0 dataset and two autism spectrum disorder curated gene sets.

G) Venn diagram of Mitocarta 2.0 dataset and the Suhl et al. curated Fmrp targets. 


\section{Supplemental Data}

Supplemental Table 1. Proteome Hits Sensitive to Activity Deprivation and Fmr1-/y Quantified by TMT Mass Spectrometry.

Supplemental Table 2. Ontological Analyses of the Proteome Sensitive to Activity Deprivation and $F m r 1^{-/ y}$. 


\section{References}

1. Bulow P, Murphy TJ, Bassell GJ, Wenner P: Homeostatic Intrinsic Plasticity Is Functionally Altered in Fmr1 KO Cortical Neurons. Cell Rep 2019, 26:1378-1388 e1373.

2. Thoby-Brisson M, Simmers J: Neuromodulatory inputs maintain expression of a lobster motor pattern-generating network in a modulation-dependent state: evidence from long-term decentralization in vitro. J Neurosci 1998, 18:2212-2225.

3. Wilhelm JC, Wenner P: GABAA transmission is a critical step in the process of triggering homeostatic increases in quantal amplitude. Proc Natl Acad Sci U S A 2008, 105:1141211417.

4. Turrigiano G: Homeostatic synaptic plasticity: local and global mechanisms for stabilizing neuronal function. Cold Spring Harb Perspect Biol 2012, 4:a005736.

5. Turrigiano G, Abbott LF, Marder E: Activity-dependent changes in the intrinsic properties of cultured neurons. Science 1994, 264:974-977.

6. Hengen KB, Lambo ME, Van Hooser SD, Katz DB, Turrigiano GG: Firing rate homeostasis in visual cortex of freely behaving rodents. Neuron 2013, 80:335-342.

7. Goold CP, Nicoll RA: Single-cell optogenetic excitation drives homeostatic synaptic depression. Neuron 2010, 68:512-528.

8. Aoto J, Nam Cl, Poon MM, Ting P, Chen L: Synaptic signaling by all-trans retinoic acid in homeostatic synaptic plasticity. Neuron 2008, 60:308-320.

9. Styr B, Gonen N, Zarhin D, Ruggiero A, Atsmon R, Gazit N, Braun G, Frere S, Vertkin I, Shapira I, et al: Mitochondrial Regulation of the Hippocampal Firing Rate Set Point and Seizure Susceptibility. Neuron 2019, 102:1009-1024 e1008.

10. Spillane M, Ketschek A, Merianda TT, Twiss JL, Gallo G: Mitochondria coordinate sites of axon branching through localized intra-axonal protein synthesis. Cell Rep 2013, 5:15641575.

11. Rangaraju V, Lauterbach M, Schuman EM: Spatially Stable Mitochondrial Compartments Fuel Local Translation during Plasticity. Cell 2019, 176:73-84 e15.

12. Soden ME, Chen L: Fragile $X$ protein FMRP is required for homeostatic plasticity and regulation of synaptic strength by retinoic acid. J Neurosci 2010, 30:16910-16921.

13. Tatavarty V, Torrado Pacheco A, Groves Kuhnle C, Lin H, Koundinya P, Miska NJ, Hengen KB, Wagner FF, Van Hooser SD, Turrigiano GG: Autism-Associated Shank3 Is Essential for Homeostatic Compensation in Rodent V1. Neuron 2020, 106:769-777 e764.

14. Blackman MP, Djukic B, Nelson SB, Turrigiano GG: A critical and cell-autonomous role for MeCP2 in synaptic scaling up. J Neurosci 2012, 32:13529-13536.

15. Lee CE, Singleton KS, Wallin M, Faundez V: Rare Genetic Diseases: Nature's Experiments on Human Development. iScience 2020, 23:101123.

16. Zhang Z, Marro SG, Zhang Y, Arendt KL, Patzke C, Zhou B, Fair T, Yang N, Sudhof TC, Wernig $\mathrm{M}$, Chen $\mathrm{L}$ : The fragile $\mathrm{X}$ mutation impairs homeostatic plasticity in human neurons by blocking synaptic retinoic acid signaling. Sci Trans/ Med 2018, 10.

17. Qiu Z, Sylwestrak EL, Lieberman DN, Zhang Y, Liu XY, Ghosh A: The Rett syndrome protein MeCP2 regulates synaptic scaling. J Neurosci 2012, 32:989-994. 
18. Wondolowski J, Dickman D: Emerging links between homeostatic synaptic plasticity and neurological disease. Front Cell Neurosci 2013, 7:223.

19. Brown V, Jin P, Ceman S, Darnell JC, O'Donnell WT, Tenenbaum SA, Jin X, Feng Y, Wilkinson $K D$, Keene JD, et al: Microarray identification of FMRP-associated brain mRNAs and altered mRNA translational profiles in fragile $X$ syndrome. Cell 2001, 107:477-487.

20. Ascano M, Jr., Mukherjee N, Bandaru P, Miller JB, Nusbaum JD, Corcoran DL, Langlois C, Munschauer $M$, Dewell $S$, Hafner $M$, et al: FMRP targets distinct mRNA sequence elements to regulate protein expression. Nature 2012, 492:382-386.

21. Darnell JC, Van Driesche SJ, Zhang C, Hung KY, Mele A, Fraser CE, Stone EF, Chen C, Fak JJ, Chi SW, et al: FMRP stalls ribosomal translocation on mRNAs linked to synaptic function and autism. Cell 2011, 146:247-261.

22. Chahrour M, Jung SY, Shaw C, Zhou X, Wong ST, Qin J, Zoghbi HY: MeCP2, a key contributor to neurological disease, activates and represses transcription. Science 2008, 320:1224-1229.

23. Cholewa-Waclaw J, Shah R, Webb S, Chhatbar K, Ramsahoye B, Pusch O, Yu M, Greulich P, Waclaw B, Bird AP: Quantitative modelling predicts the impact of DNA methylation on RNA polymerase II traffic. Proc Natl Acad Sci U S A 2019, 116:14995-15000.

24. Tillotson R, Bird A: The Molecular Basis of MeCP2 Function in the Brain. J Mol Biol 2019.

25. Lee KY, Royston SE, Vest MO, Ley DJ, Lee S, Bolton EC, Chung HJ: N-methyl-D-aspartate receptors mediate activity-dependent down-regulation of potassium channel genes during the expression of homeostatic intrinsic plasticity. Mol Brain 2015, 8:4.

26. Sutton MA, Ito HT, Cressy P, Kempf C, Woo JC, Schuman EM: Miniature neurotransmission stabilizes synaptic function via tonic suppression of local dendritic protein synthesis. Cell 2006, 125:785-799.

27. Dorrbaum AR, Alvarez-Castelao B, Nassim-Assir B, Langer JD, Schuman EM: Proteome dynamics during homeostatic scaling in cultured neurons. Elife 2020, 9.

28. Dorrbaum AR, Kochen L, Langer JD, Schuman EM: Local and global influences on protein turnover in neurons and glia. Elife 2018, 7.

29. Schanzenbacher CT, Sambandan S, Langer JD, Schuman EM: Nascent Proteome Remodeling following Homeostatic Scaling at Hippocampal Synapses. Neuron 2016, 92:358-371.

30. Gross C, Chang CW, Kelly SM, Bhattacharya A, McBride SM, Danielson SW, Jiang MQ, Chan $\mathrm{CB}$, Ye K, Gibson JR, et al: Increased expression of the PI3K enhancer PIKE mediates deficits in synaptic plasticity and behavior in fragile $X$ syndrome. Cell Rep 2015, 11:727736.

31. Muddashetty RS, Kelic S, Gross C, Xu M, Bassell GJ: Dysregulated metabotropic glutamate receptor-dependent translation of AMPA receptor and postsynaptic density-95 mRNAs at synapses in a mouse model of fragile $X$ syndrome. J Neurosci 2007, 27:5338-5348.

32. Rangaraju S, Dammer EB, Raza SA, Gao T, Xiao H, Betarbet R, Duong DM, Webster JA, Hales CM, Lah JJ, et al: Quantitative proteomics of acutely-isolated mouse microglia identifies novel immune Alzheimer's disease-related proteins. Mol Neurodegener 2018, 13:34. 
33. Wingo AP, Dammer EB, Breen MS, Logsdon BA, Duong DM, Troncosco JC, Thambisetty M, Beach TG, Serrano GE, Reiman EM, et al: Large-scale proteomic analysis of human brain identifies proteins associated with cognitive trajectory in advanced age. Nat Commun 2019, 10:1619.

34. Perez-Riverol Y, Csordas A, Bai J, Bernal-Llinares M, Hewapathirana S, Kundu DJ, Inuganti A, Griss J, Mayer $G$, Eisenacher $M$, et al: The PRIDE database and related tools and resources in 2019: improving support for quantification data. Nucleic Acids Res 2019, 47:D442-D450.

35. Kuleshov MV, Jones MR, Rouillard AD, Fernandez NF, Duan Q, Wang Z, Koplev S, Jenkins SL, Jagodnik KM, Lachmann A, et al: Enrichr: a comprehensive gene set enrichment analysis web server 2016 update. Nucleic Acids Res 2016, 44:W90-97.

36. Bindea G, Mlecnik B, Hackl H, Charoentong P, Tosolini M, Kirilovsky A, Fridman WH, Pages F, Trajanoski Z, Galon J: ClueGO: a Cytoscape plug-in to decipher functionally grouped gene ontology and pathway annotation networks. Bioinformatics 2009, 25:1091-1093.

37. Werner T, Becher I, Sweetman G, Doce C, Savitski MM, Bantscheff M: High-resolution enabled TMT 8-plexing. Anal Chem 2012, 84:7188-7194.

38. Bowling $H$, Bhattacharya A, Zhang G, Alam D, Lebowitz JZ, Bohm-Levine N, Lin D, Singha $P$, Mamcarz $M$, Puckett $R$, et al: Altered steady state and activity-dependent de novo protein expression in fragile $X$ syndrome. Nat Commun 2019, 10:1710.

39. Kuzniewska B, Cysewski D, Wasilewski M, Sakowska P, Milek J, Kulinski TM, Winiarski M, Kozielewicz $P$, Knapska $E$, Dadlez $M$, et al: Mitochondrial protein biogenesis in the synapse is supported by local translation. EMBO Rep 2020, 21:e48882.

40. Osterweil EK, Krueger DD, Reinhold K, Bear MF: Hypersensitivity to mGluR5 and ERK1/2 leads to excessive protein synthesis in the hippocampus of a mouse model of fragile $X$ syndrome. J Neurosci 2010, 30:15616-15627.

41. Bassell GJ, Warren ST: Fragile $X$ syndrome: loss of local mRNA regulation alters synaptic development and function. Neuron 2008, 60:201-214.

42. Richter JD, Bassell GJ, Klann E: Dysregulation and restoration of translational homeostasis in fragile $X$ syndrome. Nat Rev Neurosci 2015, 16:595-605.

43. Suhl JA, Chopra P, Anderson BR, Bassell GJ, Warren ST: Analysis of FMRP mRNA target datasets reveals highly associated mRNAs mediated by G-quadruplex structures formed via clustered WGGA sequences. Hum Mol Genet 2014, 23:5479-5491.

44. Zhang Y, Chen K, Sloan SA, Bennett ML, Scholze AR, O'Keeffe S, Phatnani HP, Guarnieri P, Caneda C, Ruderisch N, et al: An RNA-sequencing transcriptome and splicing database of glia, neurons, and vascular cells of the cerebral cortex. J Neurosci 2014, 34:1192911947.

45. Koopmans F, van Nierop P, Andres-Alonso M, Byrnes A, Cijsouw T, Coba MP, Cornelisse LN, Farrell RJ, Goldschmidt HL, Howrigan DP, et al: SynGO: An Evidence-Based, ExpertCurated Knowledge Base for the Synapse. Neuron 2019, 103:217-234 e214.

46. Kohler S, Carmody L, Vasilevsky N, Jacobsen JOB, Danis D, Gourdine JP, Gargano M, Harris $\mathrm{NL}$, Matentzoglu N, McMurry JA, et al: Expansion of the Human Phenotype Ontology (HPO) knowledge base and resources. Nucleic Acids Res 2019, 47:D1018-D1027. 
47. Gokhale A, Hartwig C, Freeman AAH, Bassell JL, Zlatic SA, Sapp Savas C, Vadlamudi T, Abudulai F, Pham TT, Crocker A, et al: Systems Analysis of the 22q11.2 Microdeletion Syndrome Converges on a Mitochondrial Interactome Necessary for Synapse Function and Behavior. J Neurosci 2019, 39:3561-3581.

48. Calvo SE, Clauser KR, Mootha VK: MitoCarta2.0: an updated inventory of mammalian mitochondrial proteins. Nucleic Acids Res 2016, 44:D1251-1257.

49. Jassal B, Matthews L, Viteri G, Gong C, Lorente P, Fabregat A, Sidiropoulos K, Cook J, Gillespie M, Haw R, et al: The reactome pathway knowledgebase. Nucleic Acids Res 2020, 48:D498-D503.

50. Miyashiro KY, Beckel-Mitchener A, Purk TP, Becker KG, Barret T, Liu L, Carbonetto S, Weiler IJ, Greenough WT, Eberwine J: RNA cargoes associating with FMRP reveal deficits in cellular functioning in Fmr1 null mice. Neuron 2003, 37:417-431.

51. Liu B, Li Y, Stackpole EE, Novak A, Gao Y, Zhao Y, Zhao X, Richter JD: Regulatory discrimination of mRNAs by FMRP controls mouse adult neural stem cell differentiation. Proc Natl Acad Sci U S A 2018, 115:E11397-E11405.

52. Yao A, Jin S, Li X, Liu Z, Ma X, Tang J, Zhang YQ: Drosophila FMRP regulates microtubule network formation and axonal transport of mitochondria. Hum Mol Genet 2011, 20:5163.

53. Zhang YQ, Bailey AM, Matthies HJ, Renden RB, Smith MA, Speese SD, Rubin GM, Broadie $\mathrm{K}$ : Drosophila fragile $X$-related gene regulates the MAP1B homolog Futsch to control synaptic structure and function. Cell 2001, 107:591-603.

54. Gokhale A, Lee CE, Zlatic SA, Freeman AAH, Shearing N, Hartwig C, Ogunbona O, Bassell JL, Wynne ME, Werner E, et al: Mitochondrial Proteostasis Requires Genes Encoded in a Neurodevelopmental Syndrome Locus that are Necessary for Synapse Function. bioRxiv 2020.

55. Greenblatt EJ, Spradling AC: Fragile $X$ mental retardation 1 gene enhances the translation of large autism-related proteins. Science 2018, 361:709-712.

56. Bechara EG, Didiot MC, Melko M, Davidovic L, Bensaid M, Martin P, Castets M, Pognonec $\mathrm{P}$, Khandjian $\mathrm{EW}$, Moine $\mathrm{H}$, Bardoni $\mathrm{B}$ : A novel function for fragile $\mathbf{X}$ mental retardation protein in translational activation. PLOS Biol 2009, 7:e16.

57. Chen E, Sharma MR, Shi X, Agrawal RK, Joseph S: Fragile $\mathbf{X}$ mental retardation protein regulates translation by binding directly to the ribosome. Mol Cell 2014, 54:407-417.

58. Griffiths KK, Wang A, Wang L, Tracey M, Kleiner G, Quinzii CM, Sun L, Yang G, Perez-Zoghbi $J F$, Licznerski $P$, et al: Inefficient thermogenic mitochondrial respiration due to futile proton leak in a mouse model of fragile $X$ syndrome. FASEB J 2020, 34:7404-7426.

59. Nobile V, Palumbo F, Lanni S, Ghisio V, Vitali A, Castagnola M, Marzano V, Maulucci G, De Angelis $C$, De Spirito $M$, et al: Altered mitochondrial function in cells carrying a premutation or unmethylated full mutation of the FMR1 gene. Hum Genet 2020, 139:227-245.

60. Song G, Napoli E, Wong S, Hagerman R, Liu S, Tassone F, Giulivi C: Altered redox mitochondrial biology in the neurodegenerative disorder fragile X-tremor/ataxia syndrome: use of antioxidants in precision medicine. Mol Med 2016, 22:548-559. 
61. D'Antoni S, de Bari L, Valenti D, Borro M, Bonaccorso CM, Simmaco M, Vacca RA, Catania $\mathrm{MV}$ : Aberrant mitochondrial bioenergetics in the cerebral cortex of the Fmr1 knockout mouse model of fragile $X$ syndrome. Biol Chem 2020, 401:497-503.

62. Ross-Inta C, Omanska-Klusek A, Wong S, Barrow C, Garcia-Arocena D, Iwahashi C, BerryKravis E, Hagerman RJ, Hagerman PJ, Giulivi C: Evidence of mitochondrial dysfunction in fragile X-associated tremor/ataxia syndrome. Biochem J 2010, 429:545-552.

63. Shen M, Wang F, Li M, Sah N, Stockton ME, Tidei JJ, Gao Y, Korabelnikov T, Kannan S, Vevea JD, et al: Reduced mitochondrial fusion and Huntingtin levels contribute to impaired dendritic maturation and behavioral deficits in Fmr1-mutant mice. Nat Neurosci 2019, 22:386-400.

64. Weisz ED, Towheed A, Monyak RE, Toth MS, Wallace DC, Jongens TA: Loss of Drosophila FMRP leads to alterations in energy metabolism and mitochondrial function. Hum Mol Genet 2018, 27:95-106.

65. Licznerski P, Park H-A, Rolyan H, Chen R, Mnatsakanyan N, Miranda P, Graham M, Wu J, Cruz-Reyes N, Mehta N, et al: ATP Synthase c-Subunit Leak Causes Aberrant Cellular Metabolism in Fragile X Syndrome. Cell 2020.

66. Distler U, Schumann S, Kesseler HG, Pielot R, Smalla KH, Sielaff M, Schmeisser MJ, Tenzer S: Proteomic Analysis of Brain Region and Sex-Specific Synaptic Protein Expression in the Adult Mouse Brain. Cells 2020, 9. 
A

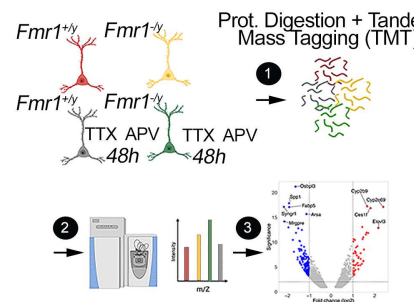

B Fmr1: $+/ y-/ y+/ y-/ y F m r 1: \quad+/ y-/ y+/ y-/ y$

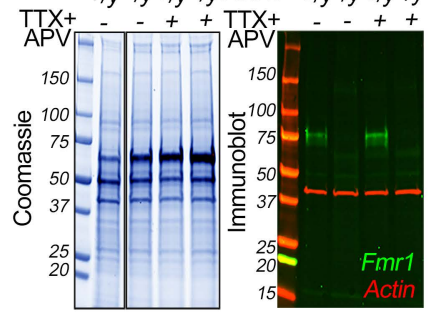

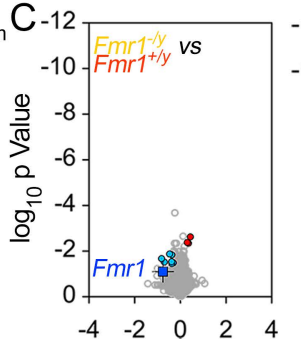
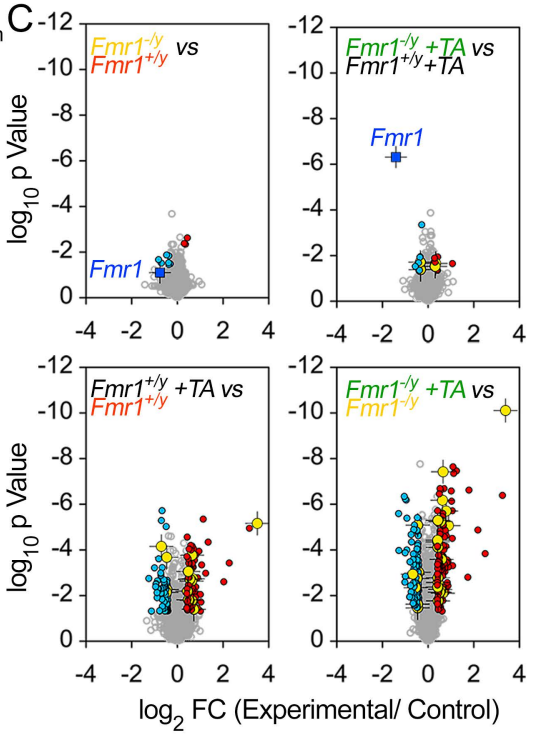

Figure 1

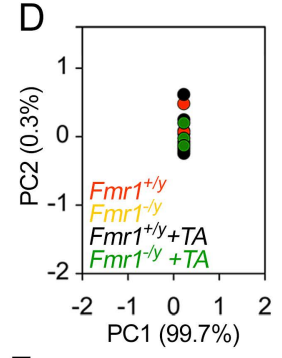

$\mathrm{F}$

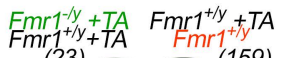

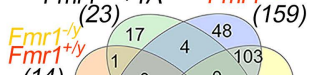

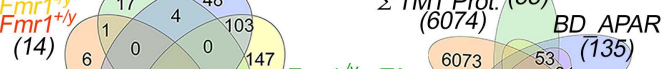
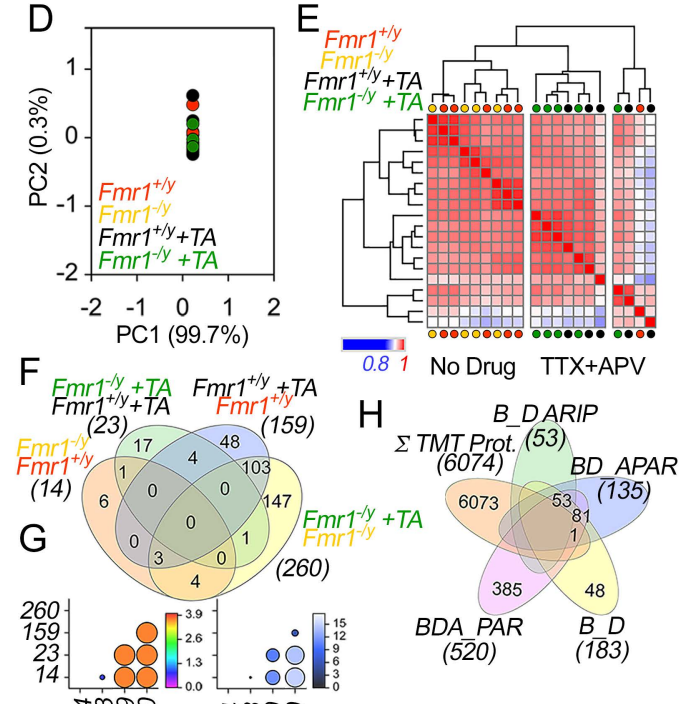

0.81 No Drug TTX+APV

$$
\mathrm{H} \quad B_{-} D A R I P
$$$$
\text { MT Prot. (53) }
$$

$$
B D \text { APAR }
$$

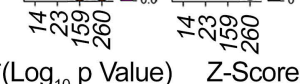

$-1^{*}\left(\log _{10} p\right.$ Value $) \quad$ Z-Score 


\section{A}

Synapse organization

Process in the synapse

Process in the postsynapse

Synaptic signaling Metabolism
Transport

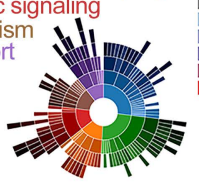

$\Sigma$ TMT Prot. (6074)

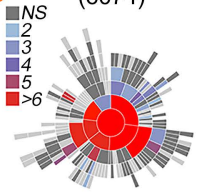

Random Geneset $\mathrm{Fmr}^{+ \text {ty }}+\mathrm{TA} \mathrm{Fmr}^{+ \text {ty }}$ (6074)

(159)
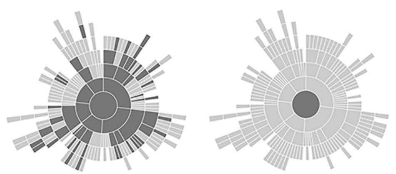

$\mathrm{Fmr1}^{-1 / y}+\mathrm{TA}$
$(260)$
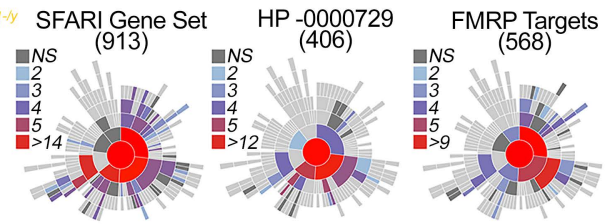

B

Fmr1-y +TA Fmr1-y

mitochondrion (GO:0005739) dendrite (GO:0030425) peroxisome (GO:0005777) microbody (GO:0042579 ribosome (GO:0005840 axon (GO:0030424) polysomal ribosome (GO:0042788) mitochondrial inner membr (GO:0005743) axonal growth cone (GO:0044295) node of Ranvier (GO:0033268)

nuclear nucleosome (GO:0000788) RNA polymerase II (GO:0090575) cytoplasmic vesicle (GO:0030659)

nuclear chromatin (GO:0000790 P-body (GO:0000932) nuclear transcription factor (GO:0044798) endoplasmic reticulum lumen (GO:0005788)

peroxisomal part (GO:0044439) main axon (GO:0044304

RNA polymerase II transcription (GO:0090575) mitochondrial inner membrane (GO:0005743)

p Value $\log 10^{*}-1$
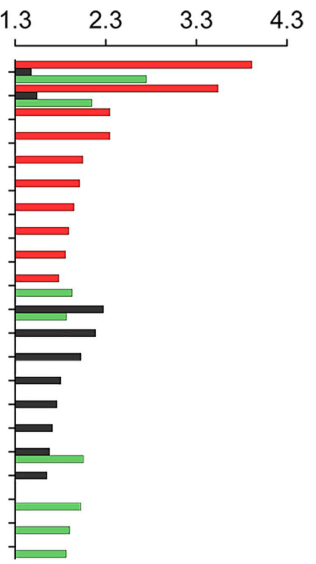

C

cytosolic part

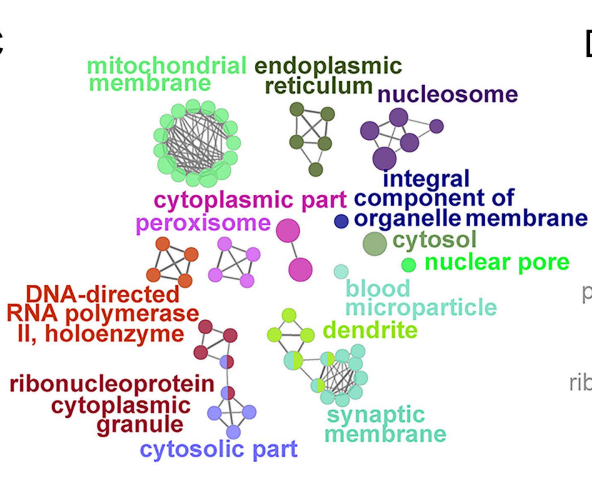

\section{D} respiratory chain
oxidoreductase complex
respiratory chain complex

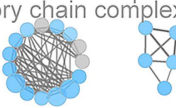

(x)

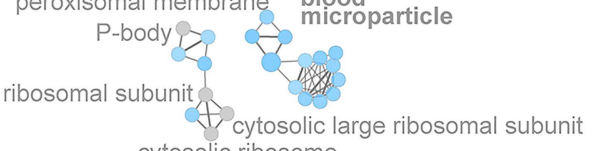

cytosolic ribosome

Equal Among
Three Clusters

$100 \%$
Distribution
Cluster 1

mitochondrial in
membrane
E

mitochondrial part

mitochondrion

mitochondrialinner endoplasmic reticulum chromatin

\section{nucleosome}
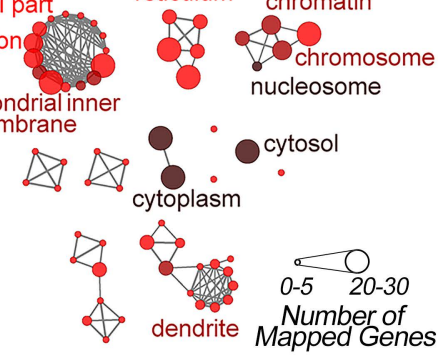

0.05 $<0.0005$ $p$ Value

\section{Figure 3}


A
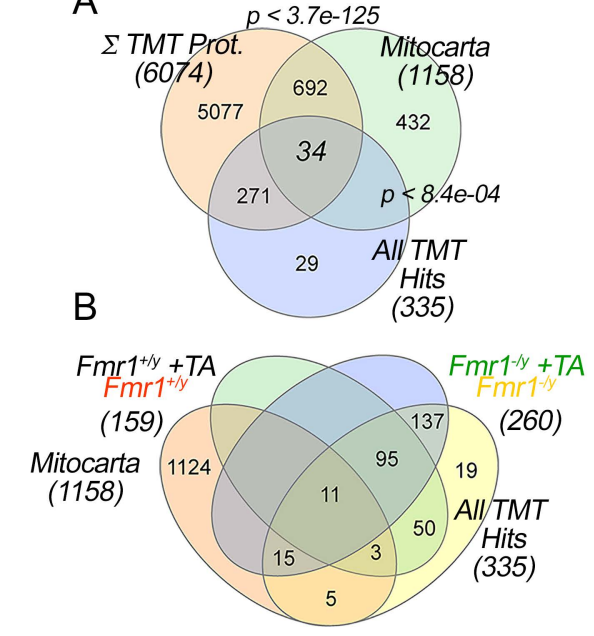

D

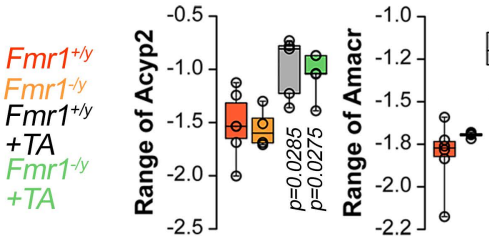

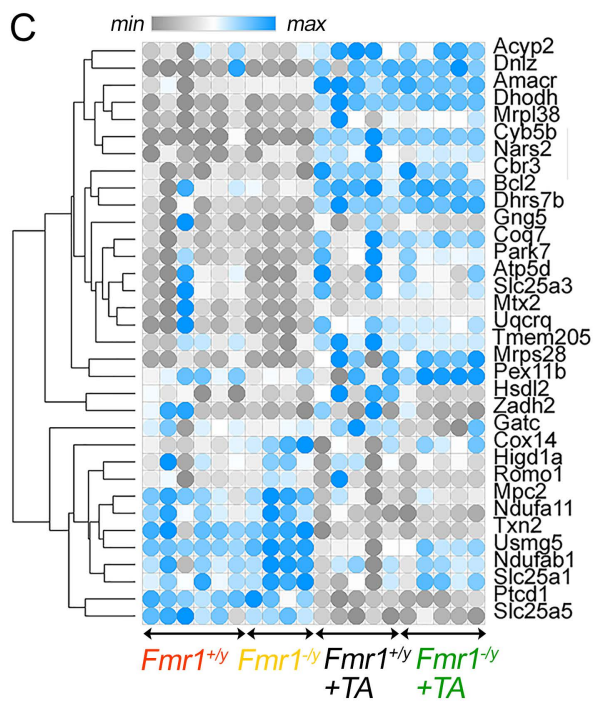

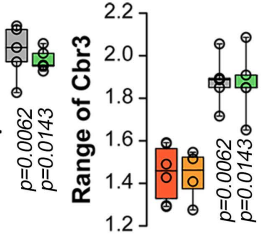

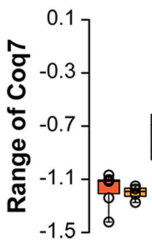

E
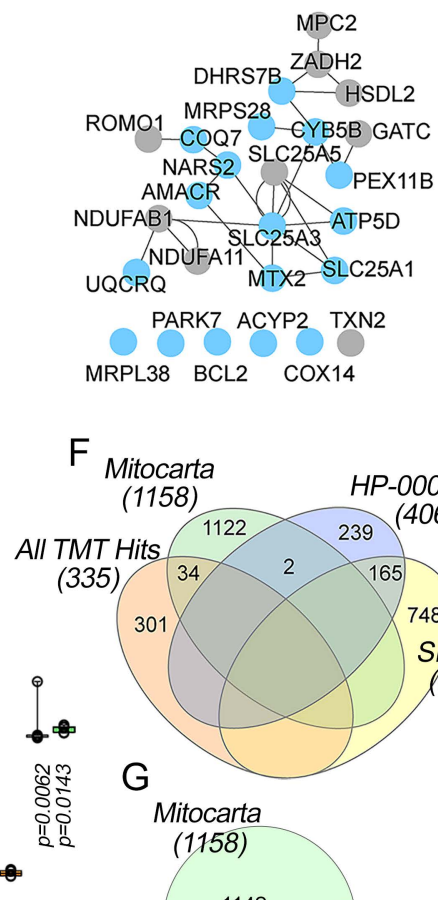

F Mitocarta

(1158)
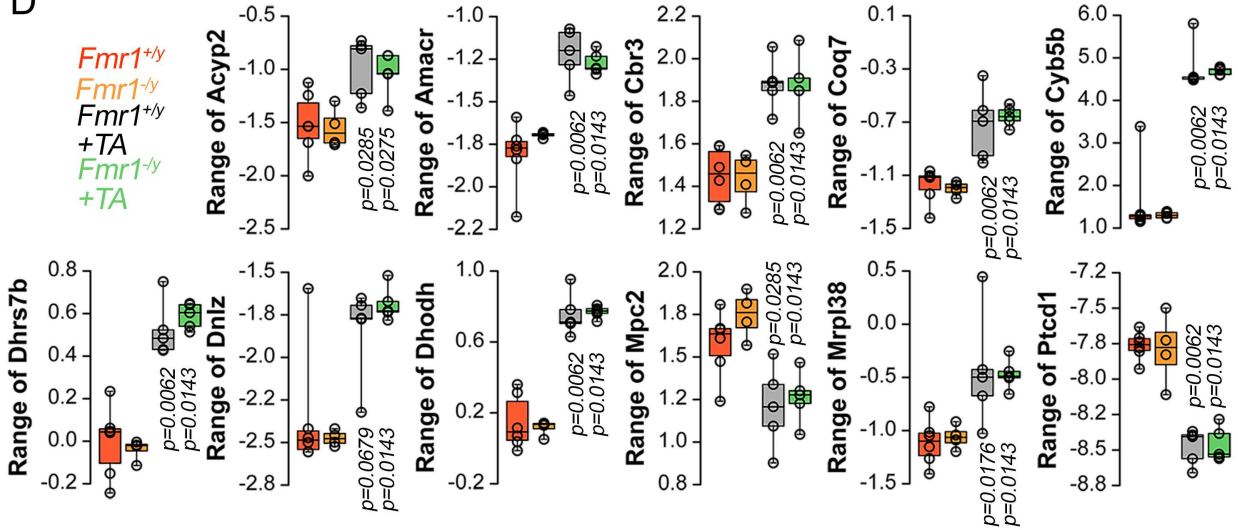

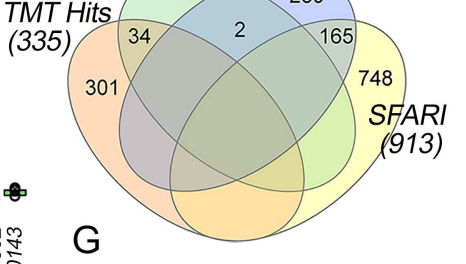

Mitocarta (1158)

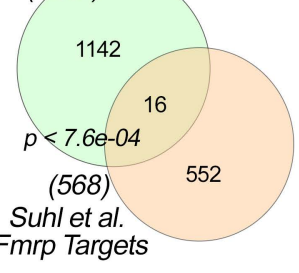

\section{Figure 4}




\section{Figures}
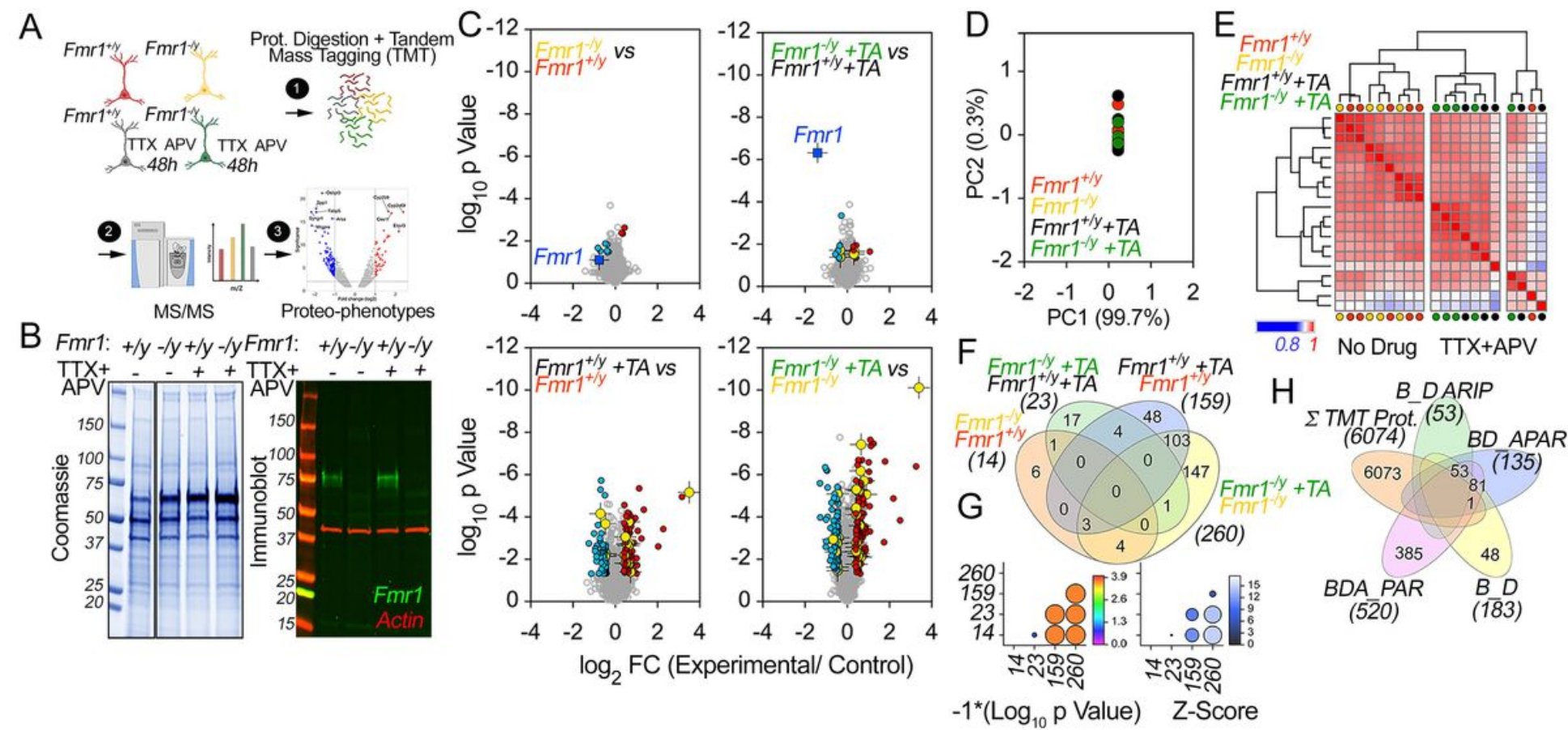

Figure 1

\section{Figure 1}

The Neuronal Proteome Sensitive to Activity Deprivation and Fmr1-/y. A) Diagram of the experimental design. B) Representative Coomassie and FMRP-beta actin blots of cultured neuronal extracts. C) Volcano Plots of TMT mass spectrometry of wild type and Fmr1-/y neurons (DIV12) incubated in vehicle or TTX-APV for 48 hours. $\mathrm{N}=6$ wild type and $n=4$ Fmr1-/y for vehicle treated cultures, $n=5$ cultures for each genotype treated with TTX-APV. Blue symbols represent downregulated proteins, red symbols upregulated proteins. FMRP is marked by dark blue and crossed square symbol. Yellow symbols depict mitochondrial proteins significantly changed. D) Principal component analysis of the 6074 TMT protein quantifications. Note close grouping of all conditions. E) Similarity matrix of wild type and Fmr1-/y neurons incubated in the presence of vehicle or TTX-APV for 48 hours. Kendall Tau clustering analysis. Note the clustering based on TTX-APV treatment. F) Venn diagram of common hits in wild type and Fmr1-/y neurons incubated in vehicle or TTX-APV for 48 hours. Parentheses represent total number of proteins significantly modified by treatment and/or genotype. Most protein changes occur after TTX-APV treatment irrespective of genotype. G) Fmr1-/y neurons incubated with TTX-APV for 48 hours significantly increase the number of hits as compared to TTX-APV-treated wild type neurons. $P$ value and $Z$ score analysis of the number of hits in $\mathrm{F} . \mathrm{X}$ and $\mathrm{Y}$ axes numbers represent the number of hits in parentheses in F). Compare wild type TTX-APV (159) to Fmr1-/y TTX-APV (260), p<0.0002 and z-score 5. Circle size and color denote $\mathrm{p}$ or z-score values. Values calculated with the Vassar's Difference between Two Independent Proportions tool. H) Venn diagram all proteins quantified by TMT overlapping with curated the FMRP target mRNAs curated by Suhl et al. 2014. See Extended Data Table 1. 
A
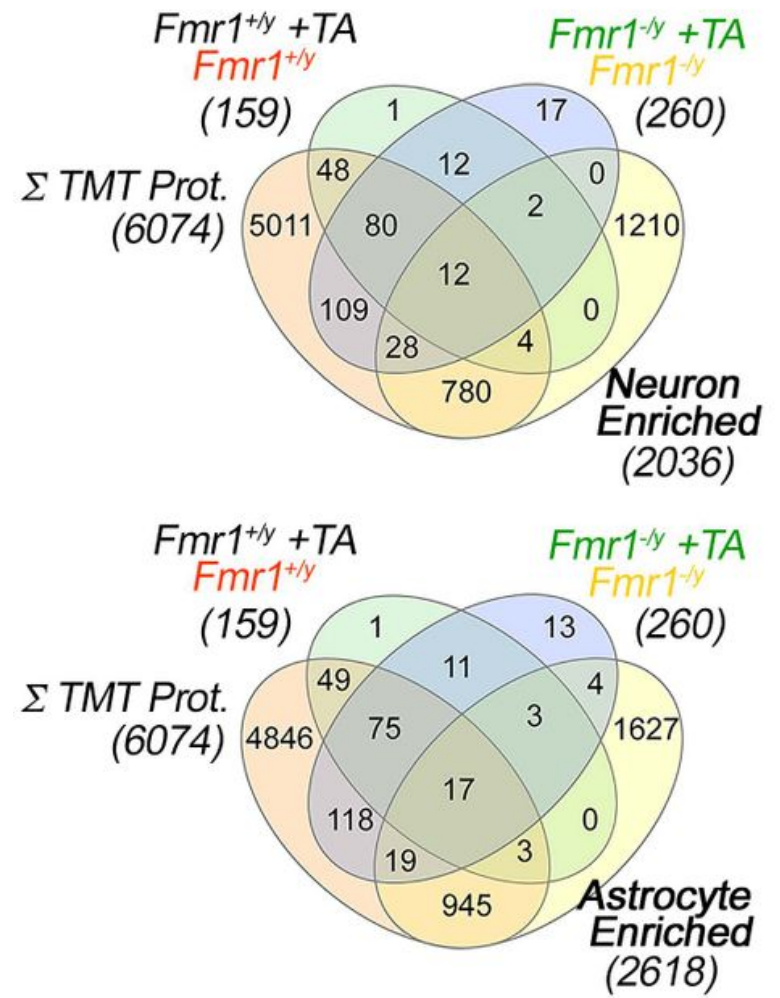

B Normalized Expression Neuron-Enriched Proteins

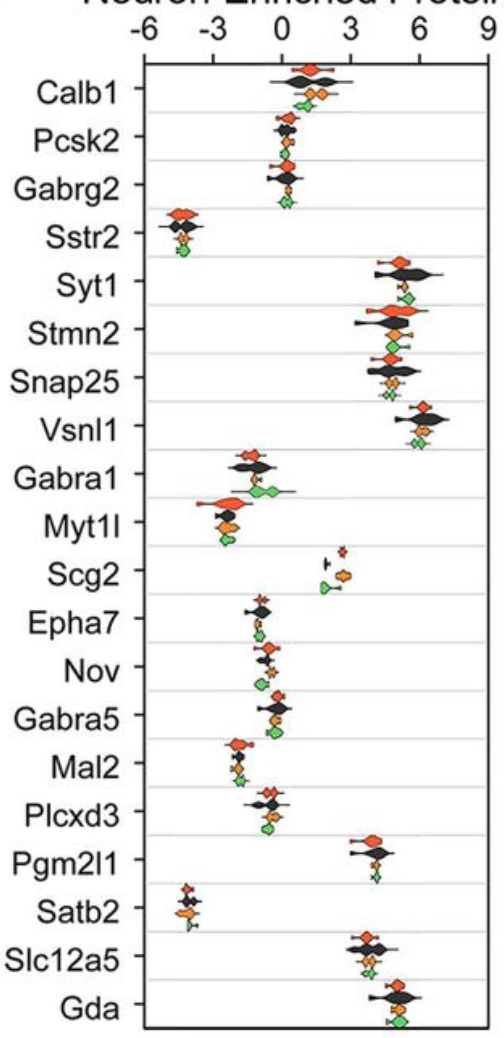

Normalized Expression Astrocyte-Enriched Proteins

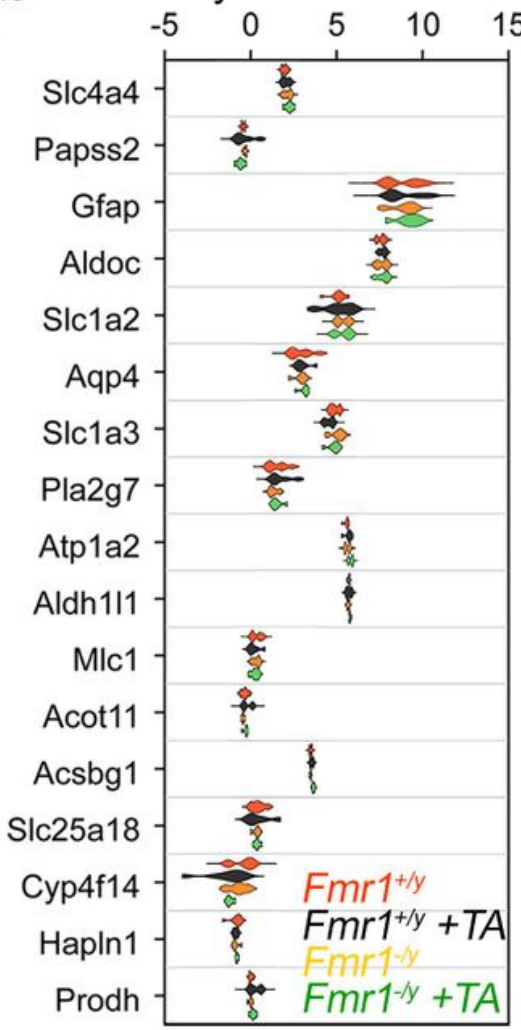

\section{Figure 2}

\section{Figure 2}

Neuronal and Glial Markers after Activity Deprivation and Fmr1 Mutation . A) Ven Diagram of overlaps between either neuronal or glial markers with all TMT quantified proteins (6074), protein hits sensitive to TTX-APV in wild type cells (159) or Fmr1-/y cells (260). Neuronal and glia enriched expressed genes were defined according to Cahoy et al. 2008 B) Violin plots depict TMT normalized expression of most abundant neuronal and astrocyte-enriched proteins in cultures treated with vehicle or TTX-APV both in wild type and Fmr1-/y genetic backgrounds. 
A

Synapse organization

Process in the synapse

Synaptic signaling

Metabolism

Transpor
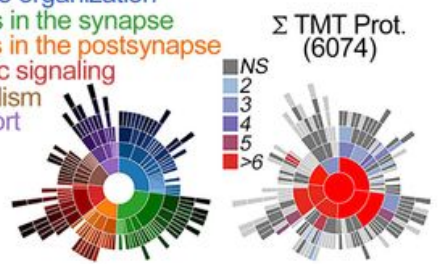

$\underset{(6074)}{R a n d o m ~ G e n}$

Geneset $F m r 1^{\text {ty }}+$ TA Fmr1+ly
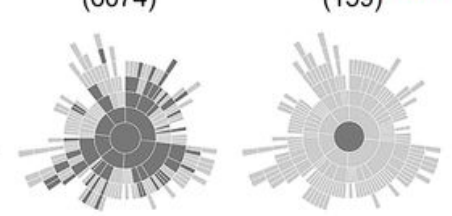

$\mathrm{Fmr}^{-1 / y}+\mathrm{TA}^{2}$
$(260)$
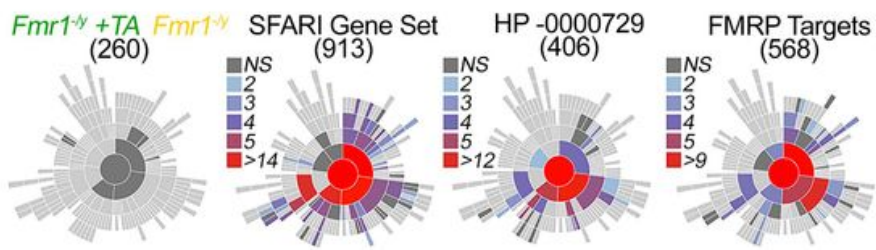

C

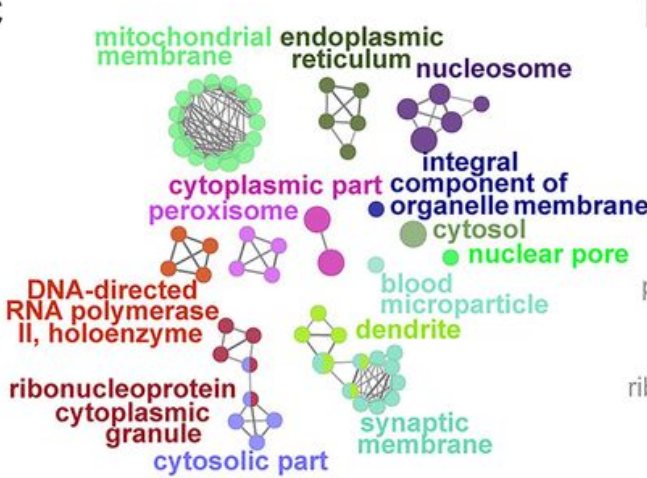

D respiratory chain respiratory chain complex
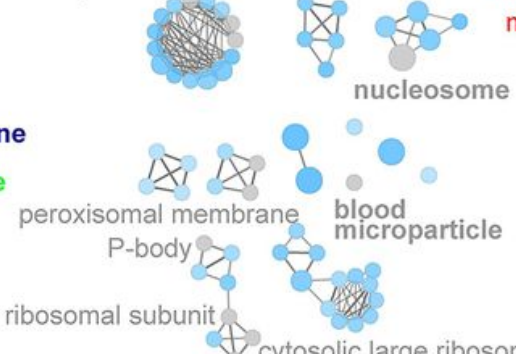

cytosolic large ribosomal subunit cytosolic ribosome

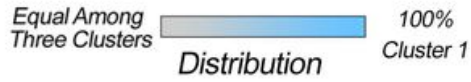

Distribution
$100 \%$
Cluster 1
All TMT Hits

$F m r 1^{+y}+T A F m r 1^{+y}$

Fmr1'y + TA Fmr1

mitochondrion (GO:0005739) dendrite (GO:0030425)

peroxisome (GO:0005777)

microbody (GO:0042579

ribosome (GO:0005840

polysomal riboson

(G):0005

axonal growth cone (GO:0044295 node of Ranvier (GO:0033268)

nuclear nucleosome (GO:0000788)

RNA polymerase II (GO:0090575

cytoplasmic vesicle (GO:0030659

nuclear chromatin (GO:0000790 P-body (GO:0000932

nuclear transcription factor (GO:0044798) endoplasmic reticulum lumen (GO:0005788) peroxisomal part (GO:0044439 main axon (GO:0044304

(G0:0090575)
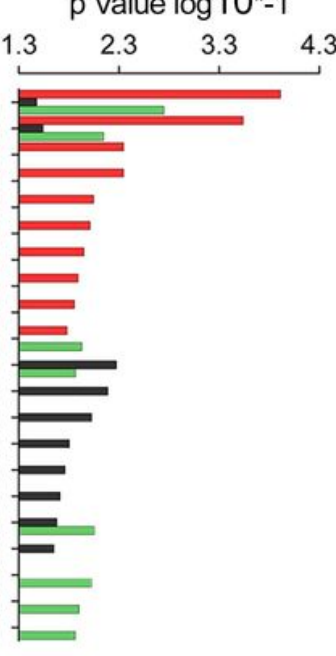
4.3

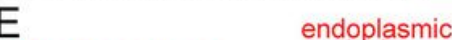

mitochondrial part reticulum chromatin mitochondrion

mitochondrial inner membrane
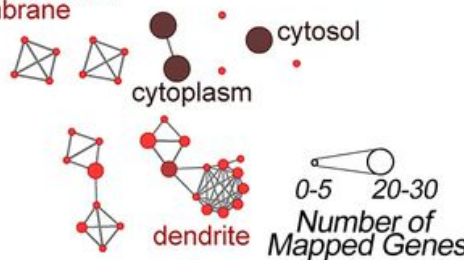

0.05

$p$ Value

Figure 3

\section{Figure 3}

Ontological Analysis of the Proteome Sensitive to Activity Deprivation and Fmr1-/y. A) Synaptic Ontology Analysis using the SYNGO tool. Sunburst plots represent synaptic annotated GO BP ontologies. Colors represent - $\log 10$ FDR corrected $p$ values. Gene sets are defined in Fig. 1. SFARI correspond to the autism spectrum disorder genes curated by https://gene.sfari.org/database/human-gene/. HP-0000729 corresponds to the Human Phenotype Ontology annotated term Autistic behavior HP:0000729 (https://hpo.jax.org/app/browse/term/HP:0000729). B) GO CC Term ontology analysis performed with the ENRICHR tool. All proteins sensitive to TTX-APV plus those sensitive to the Fmr1-/y genotype (red bars) were compared to wild type TTX-APV treated hits (black bars) and Fmr1-/y TTX-APV treated hits (green bars). C) ClueGO Ontology Analysis of all TMT Hits defined by the red bar in B. Node size represents number of mapped genes. D) ClueGO Ontology gene percentage contributions among the three gene sets in B E) ClueGO Ontology Statistics. Non-corrected Two Sided Hypergeometric Test. See Extended Data Table 2. 


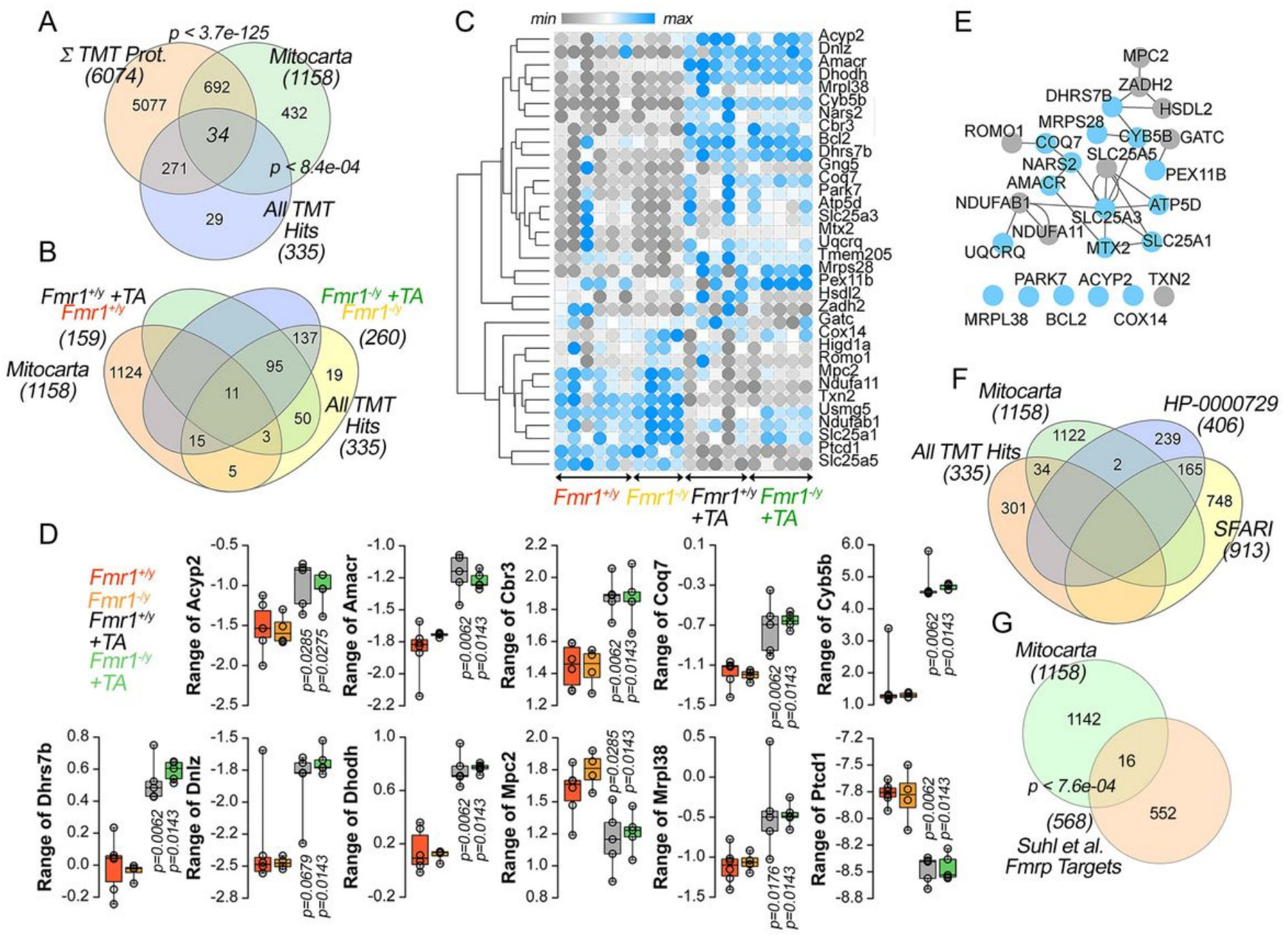

Figure 4

\section{Figure 4}

The Mitoproteome Sensitive to Activity Deprivation and Fmr1-/y. A) Venn diagram comparing all Mitocarta 2.0 entries with all TMT quantified proteins (6074), all hits sensitive to TTX-APV plus those sensitive to the Fmr1-/y genotype (335) B) Venn diagram comparing all Mitocarta 2.0 mitoproteome with protein hits sensitive to TTX-APV in wild type cells (159) or Fmr1-/y cells (260) and all TMT proteome hits (335) C) Hierarchical clustering analysis of the expression of 34 proteins in wild type and Fmr1- /y cells treated with vehicle or TTX-APV. Kendal Tau clustering analysis of rows. D) Normalized TMT expression of top mitochondrial hits. Kruskal-Wallis Test followed by Mann-Whitney U Test. $n=6$ wild type and $n=4$ Fmr1-/y for vehicle treated cultures, $n=5$ for each genotype treated with TTX-APV TTX-APV-treated. E) Interactome of the TTX-APV and Fmr1-/y sensitive mitoproteomes. Blue nodes represent upregulated proteins. Grey represent downregulated proteins. F) Venn diagram of all TMT hits, the Mitocarta 2.0 dataset and two autism spectrum disorder curated gene sets. G) Venn diagram of Mitocarta 2.0 dataset and the Suhl et al. curated Fmrp targets. 


\section{Supplementary Files}

This is a list of supplementary files associated with this preprint. Click to download.

- Table1.xlsx

- Table2.xlsx 